\title{
BRCA1 and BRCA2 Tumor Suppressor Function in Meiosis
}

\author{
Qianyan Li and JoAnne Engebrecht*
}

Department of Molecular and Cellular Biology, and Biochemistry, Molecular, Cellular and Developmental Biology Graduate Group, University of California, Davis, Davis, CA, United States

Meiosis is a specialized cell cycle that results in the production of haploid gametes for sexual reproduction. During meiosis, homologous chromosomes are connected by chiasmata, the physical manifestation of crossovers. Crossovers are formed by the repair of intentionally induced double strand breaks by homologous recombination and facilitate chromosome alignment on the meiotic spindle and proper chromosome segregation. While it is well established that the tumor suppressors BRCA1 and BRCA2 function in DNA repair and homologous recombination in somatic cells, the functions of BRCA1 and BRCA2 in meiosis have received less attention. Recent studies in both mice and the nematode Caenorhabditis elegans have provided insight into the roles of

OPEN ACCESS

Edited by: Akira Shinohara, Osaka University, Japan

Reviewed by:

Sarit Smolikove,

The University of lowa, United States

Anton Gartner,

IBS Center for Genomic Integrity, Ulsan National Institute of Science and Technology, South Korea

*Correspondence:

JoAnne Engebrecht jengebrecht@ucdavis.edu

Specialty section:

This article was submitted to Cell Growth and Division, a section of the journal Frontiers in Cell and Developmental Biology

Received: 16 February 2021 Accepted: 19 March 2021 Published: 23 April 2021

Citation:

Li Q and Engebrecht J (2021) BRCA1 and BRCA2 Tumor

Suppressor Function in Meiosis. Front. Cell Dev. Biol. 9:668309. doi: 10.3389/fcell.2021.668309 these tumor suppressors in a number of meiotic processes, revealing both conserved and organism-specific functions. BRCA1 forms an E3 ubiquitin ligase as a heterodimer with BARD1 and appears to have regulatory roles in a number of key meiotic processes. BRCA2 is a very large protein that plays an intimate role in homologous recombination. As women with no indication of cancer but carrying BRCA mutations show decreased ovarian reserve and accumulated oocyte DNA damage, studies in these systems may provide insight into why BRCA mutations impact reproductive success in addition to their established roles in cancer.

Keywords: BARD1, BRCA1, BRCA2, DSBs, meiosis, MSCI, recombination

\section{INTRODUCTION}

Homologous recombination (HR) is a high-fidelity pathway that mediates error-free repair of DNA double strand breaks (DSBs) and is essential for maintaining genome integrity. In somatic cells, DSBs can arise when DNA replication is impeded or following exposure to irradiation or other genotoxic stress. Cells deficient for HR show genomic instability including chromosome rearrangements, characteristic of most cancers (Negrini et al., 2010). In contrast to somatic cells, where DSBs pose a risk to genome integrity, during meiosis, hundreds of DSBs are purposely introduced by the topoisomerase-like protein SPO11 in early meiotic prophase and these meiotic DSBs must be accurately repaired for the production of euploid gametes (Lam and Keeney, 2014). As meiosis proceeds, meiotic DSBs are processed by DNA end resection to reveal $3^{\prime}$ overhangs (Garcia et al., 2011). The RAD51 recombinase as well as the meiosis-specific paralog DMC1 assemble on the resulting single strand DNA to form nucleoprotein filaments that mediate strand invasion and homology search for accurate repair (Shinohara and Shinohara, 2004). Meiotic DSB repair occurs concomitantly with the assembly of the synaptonemal complex (SC), the meiosis-specific multi-protein structure that forms between homologous chromosomes. In many 
organisms, SC assembly is driven by HR (Zickler and Kleckner, 2015). In the context of full length SC at the pachytene stage of meiotic prophase, a subset of recombination intermediates is processed into inter-homolog crossovers, which are essential for accurate separation of homologous chromosomes at meiosis I (Neale and Keeney, 2006; Baudat and de Massy, 2007). A large number of proteins are critical for HR, including the tumor suppressors BRCA1 and BRCA2, whose functions have been well characterized in somatic cells in the context of DNA damage and carcinogenesis. However, the roles of BRCA1 and BRCA2 during meiotic recombination have received less attention. Although the processing of DSBs by HR is similar in somatic cells and meiosis, meiotic recombination is unique in that SPO11 remains attached to the DNA end following DSB formation. Additionally, meiotic recombination occurs in the context of the SC and both sister and non-sister chromatids can serve as templates for repair. Thus, BRCA1 and BRCA2 function may be modified in meiosis to ensure accurate repair of meiotic DSBs. Studies in model organisms have provided insights into the roles of BRCA1 and BRCA2 in meiosis. This review will summarize the conserved and organism-specific meiotic functions of BRCA1 and BRCA2, focusing on recent studies in mice and C. elegans.

\section{BRCA1 IN COMPLEX WITH BARD1 IS AN E3 UBIQUITIN LIGASE CRITICAL FOR GENOME INTEGRITY}

Breast cancer susceptibility gene 1 (BRCA1) is a tumor suppressor gene, germline mutations of which are linked to familial breast and ovarian cancers (Hall et al., 1990; Futreal et al., 1994; Godwin et al., 1994; Miki et al., 1994). More than two decades of research has implicated BRCA1 function in multiple cellular pathways, including transcriptional regulation, DNA damage signaling, cell cycle checkpoints, centrosome regulation and in the repair of DNA DSBs through HR (Moynahan et al., 1999; Xu et al., 1999; Deng, 2002, 2006; Yarden et al., 2002; Caestecker and Van de Walle, 2013; Hill et al., 2014; Hatchi et al., 2015). Of critical importance, its role in promoting HR is directly linked to maintenance of genome integrity (Roy et al., 2011; Prakash et al., 2015).

In humans, the 1,863 amino acid BRCA1 protein has an N-terminal RING (Really Interesting New Gene) domain that coordinates two zinc cations in a cross-braced arrangement, a largely unstructured central region encoded by exon 11, followed by a coiled coil domain and two C-terminal BRCT repeats (Figure 1). RING domains create a platform for binding to E2 ubiquitin conjugating enzymes and facilitate the transfer of ubiquitin from the E2 to substrates, thereby specifying E3 ubiquitin ligase activity (Deshaies and Joazeiro, 2009). The BRCT repeats are phosphopeptide interaction modules for binding to phosphorylated proteins (Manke et al., 2003; Rodriguez et al., 2003; Yu et al., 2003). BRCA1 forms a heterodimer with its obligate binding partner BARD1 (BRCA1-Asssociated RING Domain protein 1) through their N-terminal regions and the heterodimer exhibits efficient ubiquitin transfer activity (Wu et al., 1996; Meza et al., 1999; Brzovic et al., 2001;
Hashizume et al., 2001; Baer and Ludwig, 2002). The BARD1 protein is 777 amino acids in length and similar to BRCA1, contains a RING domain at its N-terminus and two BRCT repeats at its C-terminus (Figure 1). In addition, four ankyrin repeats involved in chromatin recognition of newly replicated sister chromatids are present in the middle of the protein (FoxIII, Le Trong et al., 2008; Nakamura et al., 2019). Most studies indicate that BARD1 is indispensable for BRCA1 function and depletion of BARD1 leads to highly similar phenotypes as observed for BRCA1 mutants. Mutations in BARD1 have been identified in patients with breast, ovarian and other cancer types, although at a lower frequency than BRCA1 mutations (Thai et al., 1998; Ghimenti et al., 2002). Further, as with BRCA1, loss of BARD1 results in embryonic lethality in mice as well as defects in HR leading to chromosomal instability (McCarthy et al., 2003).

The mechanisms by which BRCA1-BARD1 promotes HR during DSB repair involve multiple steps. First, BRCA1 promotes DNA end resection by antagonizing 53BP1, a DNA damage response protein that promotes error-prone non-homologous end joining (NHEJ) (Bunting et al., 2010; Daley and Sung, 2014). Two, BRCA1 regulates the MRE11-RAD50-NBS1-CtIP complex essential for DNA end processing (Cruz-Garcia et al., 2014; Aparicio et al., 2016). There is also evidence that BRCA1 removes a chromatin barrier for DNA resection through ubiquitylation of histone H2A (Densham et al., 2016). In addition to promoting resection, BRCA1-BARD1 binds to DNA and interacts with RAD51 directly, enhancing RAD51 recombinase activity by promoting homologous strand invasion and synaptic complex formation (Zhao et al., 2017). However, whether BRCA1 functions by similar mechanisms to promote HR during meiosis for the repair of SPO11-induced DSBs has remained elusive.

\section{BRCA1 FUNCTION IN MOUSE MEIOSIS}

Mice homozygous for Brcal null alleles are embryonic lethal, excluding the possibility to assess BRCA1 function during meiosis (Gowen et al., 1996; Hakem et al., 1996; Liu et al., 1996; Ludwig et al., 1997). To circumvent this limitation, meiosis has been analyzed in mice carrying a hypomorphic mutation that deletes the large exon11 in the heterozygous Trp53 (encoding p53) mutant background (Brca1 $1^{\Delta 11 / \Delta 11} \operatorname{Trp53^{+/-}}$ ) (Xu et al., 2003; Figure 1). These mice develop and survive to adulthood; lethality likely bypassed by the reduced expression of $\operatorname{Tr} p 53$ (Cressman et al., 1999).

\section{BRCA1 Is Essential for Meiotic Sex Chromosome Inactivation During Spermatogenesis}

Although Trp53 heterozygosity rescues the embryonic lethality of $B r c a 1^{\Delta 11 / \Delta 11}$ mice, males are infertile as a result of pachytene arrest and apoptotic removal of germ cells (Xu et al., 2003). This observation revealed an essential role of BRCA1 in meiotic sex chromosome inactivation (MSCI). MSCI is a repressive mechanism that occurs during meiotic prophase I and involves elaboration of heterochromatin and transcriptional silencing of non-homologous regions of sex chromosomes (Turner, 2007). 


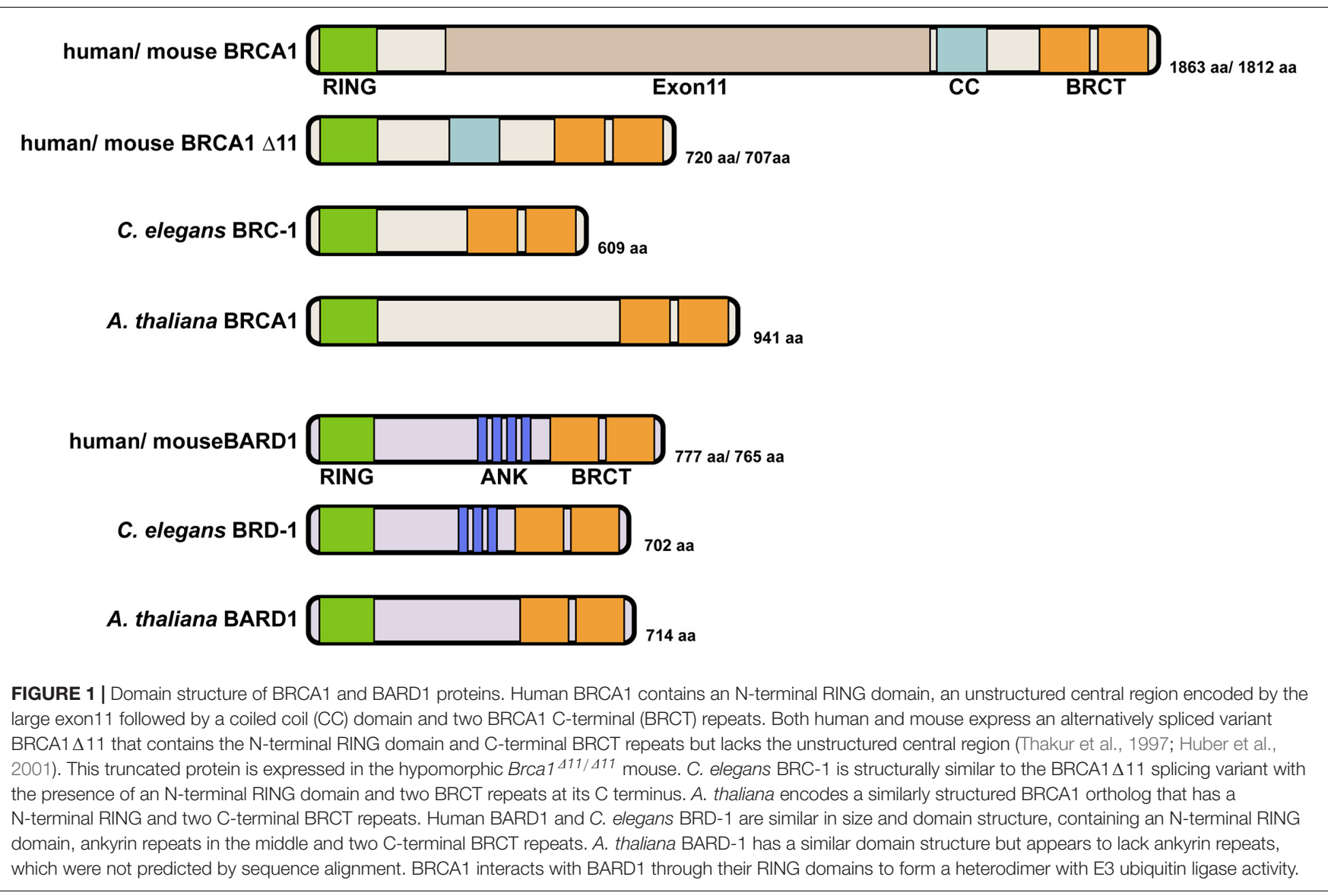

MSCI is required for efficient meiotic progression in males as failure to repress the $\mathrm{X}$ and $\mathrm{Y}$ chromosomes results in elevated germline apoptosis (Figure 2).

In wild-type spermatocytes, BRCA1 localizes to asynapsed chromosome axes, including the mostly unsynapsed $\mathrm{X}$ and $\mathrm{Y}$ sex chromosomes (Scully et al., 1997). BRCA1 recruits the checkpoint kinase ataxia telangiectasia and Rad3-related protein (ATR) to the hemizygous regions of sex chromosomes; ATR phosphorylates a histone variant, $\mathrm{H} 2 \mathrm{AX}$, to form $\gamma \mathrm{H} 2 \mathrm{AX}$, leading to sex chromosome compaction and transcriptional silencing (Fernandez-Capetillo et al., 2003; Turner et al., 2004). In the absence of full length BRCA1, ATR and $\gamma \mathrm{H} 2 \mathrm{AX}$ localization is disrupted, formation of XY sex body fails, and transcriptional silencing is abolished, leading to ectopic gene transcription from the hemizygous regions of the sex chromosomes (Xu et al., 2003; Turner et al., 2004; Broering et al., 2014). The inability to execute successful MSCI in the Brca1 $1^{\Delta 11 / \Delta 11}$ mutant has been attributed to a direct role of BRCA1 in establishing heterochromatin on the $\mathrm{X}$ and $\mathrm{Y}$ chromosomes and XY body morphogenesis, rather than an indirect consequence of defective meiotic recombinational repair in the absence of full-length BRCA1 (Broering et al., 2014).

The related process of meiotic silencing of unsynapsed chromatin (MSUC) also requires BRCA1 and operates in both male and female germ cells (Mahadevaiah et al., 2008; Kouznetsova et al., 2009). As with MSCI, MSUC leads to accumulation of repressive chromatin and transcriptional silencing on any asynapsed chromosomal regions. MSUC promotes the elimination of gametes with chromosome asynapsis and is initiated by the recruitment of BRCA1 to unsynapsed chromosomes through the interaction with the SC axial component SYCP3. Interestingly, oocytes have a limited capacity to silence unsynapsed chromosomes and this appears to be a consequence of the amount of BRCA1 available to accumulate on unsynapsed chromosomes. Thus, the role of BRCA1 in transcriptional silencing contributes to ensuring the production of euploid gametes.

\section{Potential BRCA1 Role in Meiotic Recombination}

In addition to MSCI failure, spermatocytes from $\mathrm{Brca}^{411 / \Delta 11}$ Trp53 $3^{+/-}$mice exhibited a prolonged autosomal $\gamma \mathrm{H} 2 \mathrm{AX}$ signal with greatly reduced numbers of RAD51 (but not DMC1) and MLH1 foci, suggesting that BRCA1 plays a role in meiotic DSBs repair and crossover formation (Xu et al., 2003). In contrast, a separate study utilizing Cre/LoxP mediated conditional germline-specific deletion of Brcal exon11 in the presence of both wild-type $\operatorname{Trp} 53$ alleles showed that RAD51 foci were not reduced, although decreased numbers of MSH4 foci and delayed appearance of MLH1 foci were observed. These authors concluded that while BRCA1 is not essential for meiotic DSB repair, BRCA1 might be involved in the regulation of the 


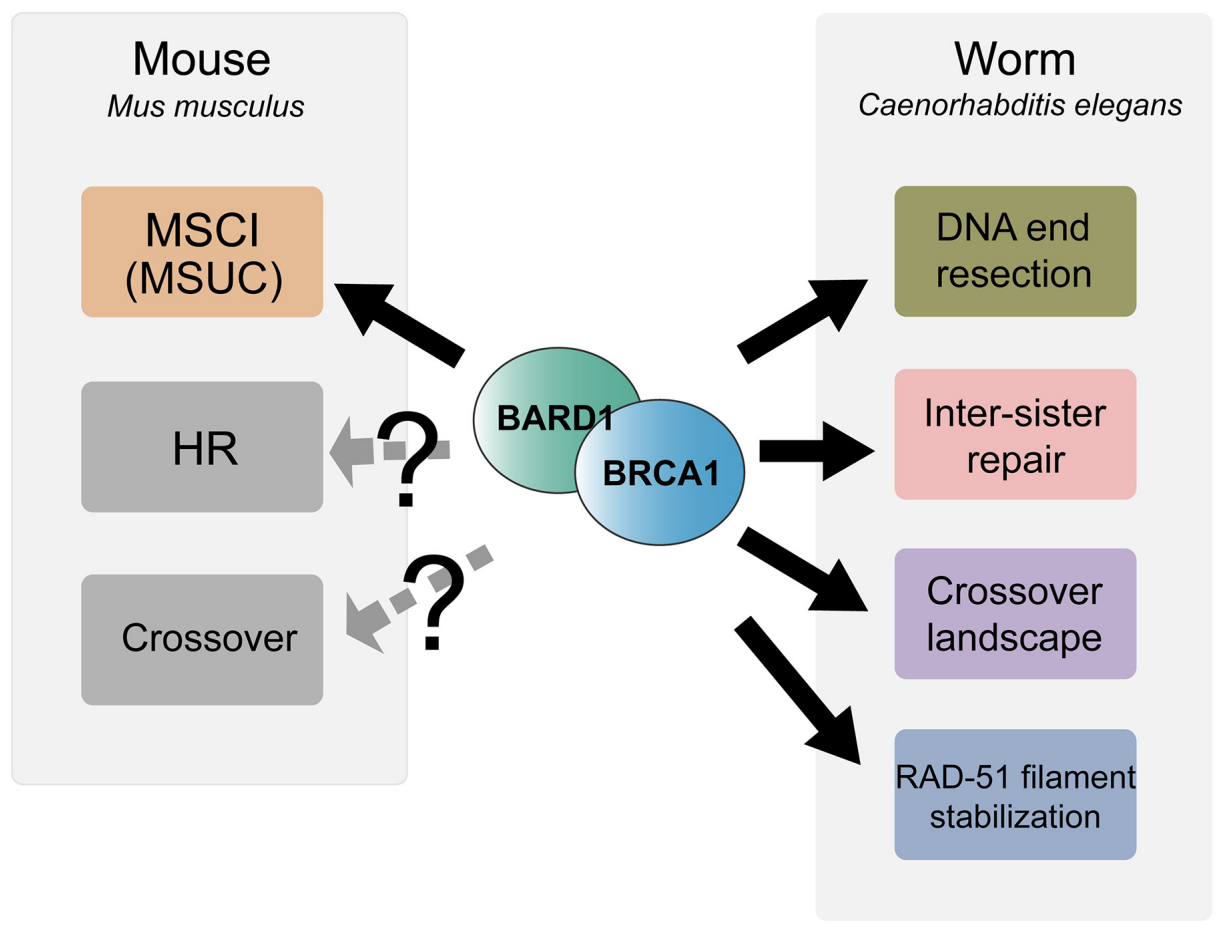

FIGURE 2 | Summary of meiotic functions of BRCA1-BARD1 in mouse and C. elegans. BRCA1-BARD1 is critical for meiotic sex chromosome inactivation (MSCI) and meiotic silencing of unsynapsed chromatin (MUSC) during mouse meiosis while it remains an open question as to whether it functions in meiotic recombination and crossover regulation. In contrast to mouse, C. elegans BRC-1-BRD-1 is dispensable for $\mathrm{MSCl}$ but functions in DNA end resection, inter-sister recombinational repair, RAD-51 filament stabilization and regulation of the crossover landscape.

timing of crossover formation (Broering et al., 2014). In a recent study using END-seq on mouse spermatocytes that allows direct examination of meiotic DSB processing at the single nucleotide level, hypomorphic Brca1 ${ }^{\Delta 11 / \Delta 11}$ Trp53 $3^{+/-}$mice did not exhibit a reduction in resection track length, suggesting that BRCA1 does not promote DNA resection in meiotic DSB repair as in somatic cells (Paiano et al., 2020). Together these results suggest that the critical meiotic role for BRCA1 is in transcriptional silencing; however, it is possible that BRCA1 function in meiotic recombination is obscured by the use of the hypomorphic Brca1 ${ }^{\Delta 11 / \Delta 11}$ mutant (Figure 2).

Analysis of female meiosis in the hypomorphic Brca1 ${ }^{411 / \Delta 11}$ mutation revealed no observable phenotypes. Female Brca1 mutants are fertile and the number of MLH1 foci are comparable to that observed in wild-type oogenesis, suggesting that the region deleted in $B r c a 1^{\Delta 11 / \Delta 11}$ is not required for meiotic recombination during female meiosis (Xu et al., 2003; Broering et al., 2014). Therefore, the observed sex-specific phenotypes in the hypomorphic Brca1 $1^{\Delta 11 / \Delta 11}$ mutant are likely a consequence of the presence of unsynapsed sex chromosomes in males. It is also important to note that the region encoded by exon 11 is thought to be unstructured with no resemblance to known domain structures ( $\mathrm{Li}$ and Greenberg, 2012). Future studies focusing on the RING domain, which confers E3 ubiquitin ligase activity, and the BRCT repeats, are necessary to reveal whether these domains play important roles in the repair of meiotic DSBs in both male and female meiosis. Finally, to the best of our knowledge a functional role of BARD1 in mice gametogenesis has not been examined.

\section{BRCA1 FUNCTION IN C. ELEGANS MEIOSIS}

\section{The C. elegans Germ Line as a Model for Studying Meiosis and BRCA1-BARD1 Function}

Caenorhabditis elegans has emerged as an excellent model for investigating meiosis: many genes required for meiotic recombination are conserved in this metazoan and the animals possess prominent gonads that exhibit a spatial temporal organization of germ cells undergoing meiotic prophase I (Figure 3A). At the distal tip, germline stem cells divide to produce cells that will advance down the gonad and enter meiosis. In transition zone (corresponding to leptotene/zygotene), homologous chromosomes are paired together, facilitated by Zn-finger ZIM-1/2/3 and HIM-8 proteins that bind to special sequences present on each homolog pair. Beginning at this stage, SPO-11 induces meiotic DSBs, which are processed and bound by $\mathrm{RAD}-51$ for homologous recombinational repair. In pachytene, the SC is fully assembled between the homologs and within this context strictly one crossover forms between each chromosome pair in late pachytene. Upon crossover formation, the SC 
disassembles and homologs undergo remodeling and compaction to reveal six bivalents at diakinesis stage, representing the six pairs of homologs connected by chiasmata (Figure 3B; Lui and Colaiacovo, 2013; Hillers et al., 2017). Although the overall process is very similar to other systems, it is important to note that there are differences unique to $C$. elegans meiosis. These include the absence of DMC1 in this organism, thus RAD51 is the sole recombinase acting during both mitotic and meiotic recombination (Brown and Bishop, 2014). Interestingly, C. elegans RAD-51 contains three amino acids conserved in the DMC1 lineage that stabilize mismatch-containing heteroduplex DNA, critical for meiotic recombinase function (Steinfeld et al., 2019). Another unique feature of C. elegans meiosis is that chromosome synapsis does not depend on meiotic recombination initiation (Dernburg et al., 1998). Nevertheless, the availability of molecular markers combined with genetic and genomic approaches has made the C. elegans germ line a powerful system that provides a unique opportunity to dissect gene function at any particular sub-stage of meiotic prophase. Most importantly, proteins with conserved domain structure and sequence similarity to BRCA1 and BARD1, referred to as BRC-1 and BRD-1, are encoded in the C. elegans genome. brc-1 and brd-1 null mutants exhibit elevated IR sensitivity and a higher incidence of males among self-progeny (a readout of $\mathrm{X}$ chromosome non-disjunction) compared to wild type, but are mostly fertile, allowing analysis of meiotic outcomes in the absence of functional BRCA1 and BARD1 (Boulton et al., 2004; Li et al., 2018). Similar to C. elegans, Arabidopsis AtBRCA1 and AtBARD1 mutants are also fertile, suggesting that the essentiality of mammalian BRCA1-BARD1 is not broadly conserved (Reidt et al., 2006).
C. elegans brc-1 encodes a 609 amino acid protein with highly conserved N-terminal RING domain and C terminal BRCT repeats, similar to the human protein. Structurally, C. elegans BRC- 1 is analogous to the BRCA $1 \Delta 11$ splicing variant (Figure 1). AtBRCA1 with 941 amino acids is also considerably smaller than the human protein. The C. elegans BRD-1 and AtBARD1 proteins are similar in both size and domain architecture to the human protein, although AtBARD1 does not have recognizable ankyrin repeats (Figure 1). Interestingly, C. elegans BRC-1-BRD1 exhibits dynamic localization throughout meiotic prophase. Discrete foci of BRC-1-BRD-1 that partially colocalize with RAD-51 are present in both proliferative/mitotic region and early meiotic prophase, from leptotene to early pachytene ( $\mathrm{Li}$ et al., 2018, 2020). As meiotic prophase progresses, BRC-1BRD-1 localizes with the SC between the maternal and paternal chromosomes (Polanowska et al., 2006; Janisiw et al., 2018; Li et al., 2018). This localization is in contrast to BRCA1 localization in mammalian meiocytes, where BRCA1 is found on the axes of asynapsed chromosomes (Turner et al., 2004). In late pachytene upon crossover maturation, BRC-1-BRD1 concentrates on one subdomain of the chromosome pair termed the "short arm", suggesting an intimate connection of BRC-1-BRD-1 to crossover sites and potential involvement in crossover regulation.

\section{BRC-1-BRD-1 Is Not Essential for Meiotic Sex Chromosome Inactivation but Promotes HR in Spermatogenesis}

C. elegans BRC-1-BRD-1 is absent from the single asynapsed $\mathrm{X}$ chromosome in male germ cells, and consistent with

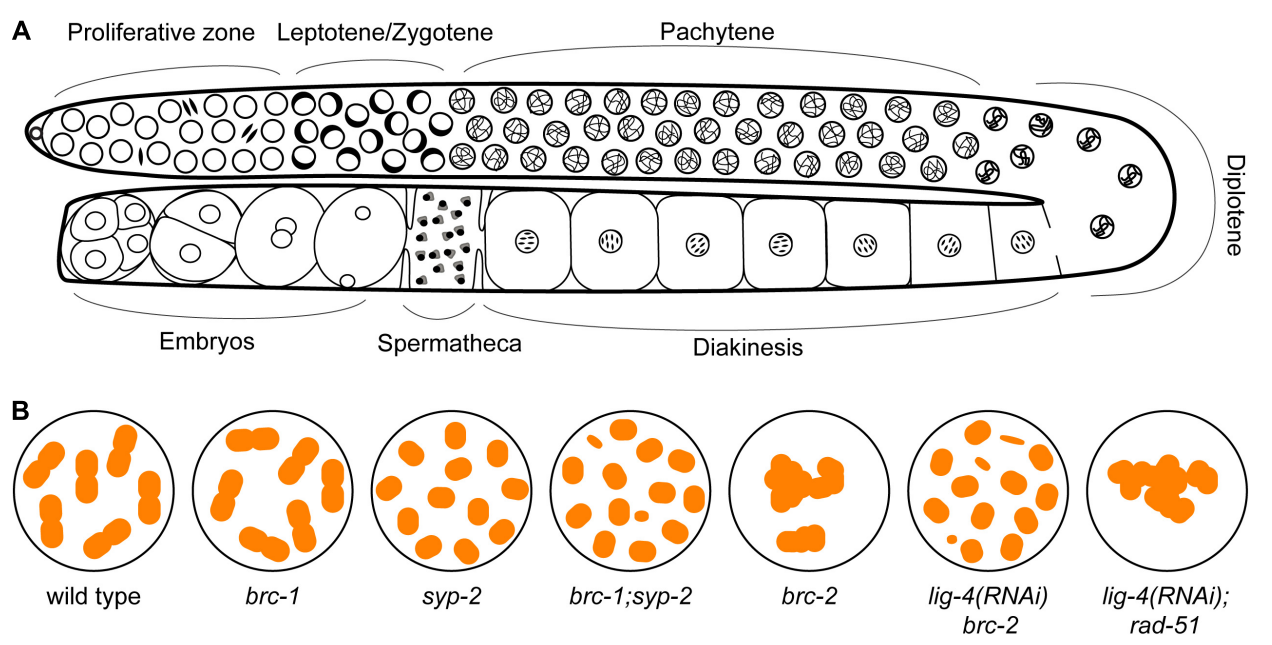

FIGURE 3 | The C. elegans germ line presents a spatial temporal organization of events during meiotic prophase I. (A) At the distal proliferative zone, germline stem cells mitotically divide to produce cells that will advance down the gonad and enter meiosis. Chromosome pairing and DSBs induction by SPO-11 occur in leptotene/zygotene (transition zone), which is characterized by the presence of clustered chromatin on one side of the nuclei. DSBs are processed and bound by RAD-51 for homologous recombinational repair, which are visible as RAD-51 foci by immunostaining starting in leptotene through pachytene. The synaptonemal complex (SC) is fully assembled between the homologs in pachytene and strictly one crossover forms between each chromosome pair in late pachytene. Upon crossover formation, the SC disassembles and homologs undergo remodeling and compaction to reveal six bivalents at diakinesis stage. (B) Cartoon of chromosome structure observed in diakinesis nuclei in WT (6 bivalents), brc-1 (6 bivalents), syp-2 (12 univalents), brc1; syp-2 (> 12 univalents/DNA fragments) (Adamo et al., 2008), brc-2 (aggregation), lig-4 (RNAi) brc-2 (12 univalents with some DNA fragments) and lig-4 (RNAi); rad-51 (aggregation) (Martin et al., 2005) mutants. 
this observation, BRC-1-BRD-1 is not required for MSCI during spermatogenesis. In $b r c-1$ and $b r d-1$ null mutants, deposition of the repressive chromatin mark H3K9me2 and the absence of Pol2-S2P (actively transcribing RNA polymerase II) signal on the $\mathrm{X}$ chromosome are indistinguishable from wild-type animals, suggesting that MSCI is successful in these mutants. As such, the null mutants do not exhibit pachytene arrest and germ cells complete meiotic prophase in preparation for the meiotic divisions (Li et al., 2020).

Analysis of RAD-51 immunostaining in the brc-1 and brd1 null male germ lines showed reduced levels of RAD-51 foci in early meiotic prophase and this reduction was suppressed by inhibiting the NHEJ pathway. Moreover, quantification of GFP:RPA-1 foci, indicative of single stranded DNA, showed a significant reduction in overall foci number and intensity in the absence of BRC-1-BRD-1, suggesting that BRC-1-BRD-1 favors $\mathrm{HR}$ at the expense of NHEJ through promoting resection of DSBs during male meiosis ( $\mathrm{Li}$ et al., 2020; Figure 2). This role is similar to what is proposed for BRCA1 function in promoting HR in somatic cells.

\section{BRC-1-BRD-1 Promotes Inter-Sister Recombination and Stabilizes the RAD-51 Filament Under Checkpoint Activation in Oogenesis}

In contrast to male meiosis, $b r c-1$ and $b r d-1$ null mutants exhibited an increased number of RAD-51 foci at late pachytene in oogenic germ lines, with no obvious difference in RAD51 kinetics in early meiotic prophase as compared to wildtype animals (Adamo et al., 2008; Janisiw et al., 2018; Li et al., 2018). The elevated RAD-51 foci observed in late pachytene suggests that the repair of a subset of DSBs is delayed in the absence of BRC-1-BRD-1. The high fertility and presence of six bivalents, representing the six homologs connected by chiasmata, at diakinesis in brc-1 and brd-1 mutants (Figure 3B) suggest that BRC-1-BRD-1 is not essential for crossover formation. To test the hypothesis that BRC1 promotes repair of DSBs by the inter-sister recombination pathway, Adamo and coworkers disrupted SC assembly and thereby inter-homolog crossovers by mutation of syp-2 (one of six components in the central region of the SC) in the brc-1 mutant. syp-2 mutants have twelve intact univalents at diakinesis (Figure 3B), suggesting efficient repair of DSBs by the intersister pathway. On the other hand, in the brc-1; syp-2 double mutant more than twelve DAPI staining bodies were often observed (Figure 3B), indicating the presence of chromosome fragmentation and failure in inter-sister repair. These results are consistent with BRC-1 playing an important role in intersister repair during oogenesis (Adamo et al., 2008). A recent study extended these findings by showing that mutation of brc1 enhanced the phenotype of phosphorylation defective mutants in syp-1 (another component of the central region of the SC), presumably through impairment of inter-sister recombination (Garcia-Muse et al., 2019; Figure 2). Importantly, BRC-1dependent inter-sister repair prevents erroneous recombination (recombination between heterologous sequences) in meiosis, suggesting one mechanism by which $\mathrm{BRC}-1$ prevents genome instability (Leon-Ortiz et al., 2018).

In addition to promoting inter-sister repair, BRC-1 is required to stabilize the RAD-51 filament from premature disassembly in late pachytene under meiotic checkpoint activation conditions. In zim-1/2/3 or syp- 1 mutants, which lack crossovers on a subset or all chromosomes, respectively, and activate meiotic checkpoints, extensive RAD-51 foci are present throughout meiotic prophase (Yu et al., 2016). Removing BRC-1 in these mutant backgrounds results in a region in late pachytene with significantly reduced RAD-51 levels, with high levels of RAD51 both prior to and after this region. Both the number of RAD-51 foci as well as the fluorescence intensity of residual foci was greatly diminished in this region and thus this pattern has been referred to as a RAD-51 "dark zone". Taking advantage of the spatial temporal organization of the germ line, time course analysis of $s p o-11 ; b r c-1 ; s y p-1$ mutants exposed to irradiation (IR) was performed. The spo-11 mutant was used so that breaks could be induced uniformly in the germ line at a single point in time by IR and as nuclei moved through the germ line no new breaks were formed. This analysis revealed that RAD-51 installed on processed DSBs in nuclei residing in early prophase at the time of DSB induction was dismantled once the nuclei reached late pachytene, suggesting that BRC1 promotes the stability of the RAD-51 filament under these conditions (Li et al., 2018). The mechanism underlying BRC1-dependent RAD-51 stabilization is currently unknown and could be either through direct interaction with RAD-51 to reduce its ATP hydrolysis and/or regulation of helicases which dismantle the RAD-51 filament. Interestingly, the requirement for BRC-1 to stabilize RAD-51 filaments under checkpoint activation conditions is oogenesis-specific, as a RAD-51 dark zone was not observed in the male germ line (Li et al., 2020; Figure 2).

Recent studies examining the mutational signatures of $b r c-1$ and $b r d-1$ mutants propagated over multiple generations revealed elevated levels of small deletions, deletions-insertions, single nucleotide variants and tandem repeats (Kamp et al., 2020; Volkova et al., 2020). Analysis of $b r c-1$ and $b r d-1$ mutants in combination with mutations in different repair pathways provided evidence that theta-mediated end joining (TMEJ), but not NHEJ, was responsible for the mutational profiles observed. TMEJ anneals short regions of microhomology and catalyzes template-dependent DNA synthesis to repair the broken DNA molecule. These results suggest that in the absence of BRC-1-BRD-1, TMEJ repairs inefficiently resected DSBs. It will be important to distinguish whether the mutations are a consequence of repair of meiotic DSBs, or repair of breaks generated during replication prior to meiotic entry or during embryogenesis, to understand the complete spectrum of BRC-1-BRD-1 function in both the soma and in meiosis. Nonetheless, the mutational profile of C. elegans brc-1 and brd1 mutants is very similar to that found in BRCA1-deficient tumor cells, suggesting that TMEJ repair in the absence of BRCA1 contributes to carcinogenesis (Kamp et al., 2020; Volkova et al., 2020). 


\section{BRC-1-BRD-1 Regulates Crossover Patterning}

Given that there are many more DSBs than crossovers, a subset of processed DSBs is chosen to be resolved as crossovers in a process referred to as crossover designation (Gray and Cohen, 2016). To investigate whether BRC-1 plays a role in crossover designation and/or resolution, genetic linkage analysis on meiotic products of brc-1 mutants was performed and revealed an altered crossover landscape. Although the genetic map length was not significantly different between wild type and brc-1 mutants, there was a shift in crossover distribution from chromosome arms, which are most often observed in wild-type animals, to more central regions on chromosomes (Li et al., 2018, 2020). Altered crossover distribution to the chromosome center has been observed in many other C. elegans mutants defective for various aspects of meiotic recombination (Zetka and Rose, 1995; Wagner et al., 2010; Meneely et al., 2012; Saito et al., 2012, 2013; Chung et al., 2015; Hong et al., 2016; Jagut et al., 2016). While the underlying mechanisms are currently unknown, it has been suggested that this could result from an altered chromatin landscape (Saito and Colaiacovo, 2017). Thus, BRCA1 may regulate chromatin structure in C. elegans meiosis, as it does in mouse meiosis (Broering et al., 2014; Densham et al., 2016), although the specific types of chromatin modification regulated by BRCA1 may not be identical in C. elegans and mouse.

Surprisingly, in the zim-1 mutant where two chromosomes fail to pair and synapse, BRC-1-BRD-1 promoted the formation of extra COSA-1 marked crossover designation events on the remaining chromosome pairs during oogenesis. COSA-1 (Cross $\underline{\text { Over }}$ Site Associated protein 1) is generally accepted to mark canonical crossovers in C. elegans meiosis (Yokoo et al., 2012); therefore, the number of COSA-1 foci has been used as a cytological readout of the number of genetic crossovers. The reduced COSA-1 foci in the brc-1; zim-1 double mutant, however, was not accompanied by a smaller genetic map distance, measured by SNP marker-based linkage analysis. These results suggest that not all crossovers are marked by COSA-1 in the brc-1; zim-1double mutant. Further, while the map length was similar in the absence of BRC-1, CO patterning was altered such that there were elevated levels of single crossovers (SCOs) with a concomitant reduction in double crossovers (DCOs). As a crossover can form between any two non-sister chromatids within paired homologs, two, three or four-strand DCOs are possible outcomes of elevated crossover formation. However, only DCOs between the same two chromatids can be detected as DCOs in SNP marker-based analysis, because only one sister chromatid is inherited in the product of meiosis. DCOs involving three or four chromatids will be detected as SCOs. Therefore, the aforementioned observation is consistent with a model whereby inactivation of BRC-1 in the zim-1 mutant results in a shift from two-strand DCOs that are marked by COSA-1 and observed in the DCO class, to three- and four-strand DCOs that lack the COSA-1 marker and are detected as SCOs (Li et al., 2018). In contrast to oogenesis, BRC-1 inhibits the formation of extra COSA-1 marked crossover precursors in spermatogenesis. Elevated levels of COSA-1 foci were observed in the brc-1; zim-1 double mutant as compared to zim-1. Additionally, the genetic map distance was enlarged in the brc- 1 ; zim-1 double mutant, suggesting that BRC-1 inhibits the formation of extra canonical crossovers in spermatogenesis (Li et al., 2020). Together, these results suggest that $\mathrm{BRC}-1$ plays a role in $\mathrm{CO}$ patterning, perhaps through regulating both canonical and non-canonical $\mathrm{CO}$ pathways under conditions of meiotic dysfunction (Figure 2).

Why does $b r c-1$ and $b r d-1$ mutation exhibit sex-specific phenotypes? One hypothesis is that BRC-1-BRD-1 interacts with unique partners to form different complexes during male and female meiosis. This would be analogous to what has been established for BRCA1 function in somatic cells, where it forms three different complexes with distinct functions under different physiological conditions (Li and Greenberg, 2012). Alternatively, or in addition, the sex-specific phenotypes could be a consequence of BRC-1-BRD-1 being differentially regulated by post-translational modifications in the diverging environments of male and female meiosis. Future studies on BRC-1-BRD1 interacting proteins and the regulation of complex(es) will provide insight into the functions of BRC-1-BRD-1 during spermatogenesis and oogenesis. These studies may also shed light on the sex-specific regulation of the BRCA1-BARD1 complex in mammals.

\section{BRCA2 FUNCTIONS AS AN ESSENTIAL MEDIATOR FOR HR}

Breast cancer susceptibility gene 2 (BRCA2) is an essential mediator of HR (Jensen et al., 2010; Liu et al., 2010; Kowalczykowski, 2015). Similar to BRCA1, germline mutations in $B R C A 2$ predispose patients to breast and ovarian cancer and genome instability (Wooster et al., 1995; Yu et al., 2000; Venkitaraman, 2002; King et al., 2003). Biochemical, cell biological and genetic studies have supported a role of BRCA2 in recruiting the RAD51 recombinase to resected single strand DNA at DSBs and promoting nucleoprotein filament assembly to mediate homology search and strand exchange (Sharan et al., 1997; Wong et al., 1997; Abbott et al., 1998; Chen et al., 1999; Tutt et al., 1999; Yuan et al., 1999; Moynahan et al., 2001; Xu et al., 2001; Jensen et al., 2010; Liu et al., 2010; Thorslund et al., 2010).

Human BRCA2 encodes an exceptionally large protein consisting of 3,418 amino acids with multiple functional domains: an N-terminal domain that facilitates binding with $\underline{\text { Partner }} \underline{\text { And }} \underline{\text { Localizer of }} \underline{\mathrm{BRCA}} \underline{2}$ (PALB2), eight BRC repeats that define the RAD51 binding motif, a DSS1 and DNA binding domain (DBD, composed of one helix-rich domain (HD), three oligonucleotide/oligosaccharide binding (OB) folds and a tower domain), and a $\mathrm{C}$ terminal RAD51 binding domain (CTRB) (Figure 4; Yang et al., 2002; Esashi et al., 2005; Xia et al., 2006; Carreira et al., 2009; Shivji et al., 2009). Given its essential role in $\mathrm{HR}$, it is not surprising that BRCA2 is conserved in fungi, plants and metazoans. While overall similar, BRCA2 orthologs possess different numbers of BRC repeats and OB folds, which are signature domains of BRCA2, and vary considerably in size (Gudmundsdottir and Ashworth, 2004; Figure 4). For example, Brh2, the BRCA2 ortholog in the fungus Ustilago maydis, contains a single BRC repeat and 

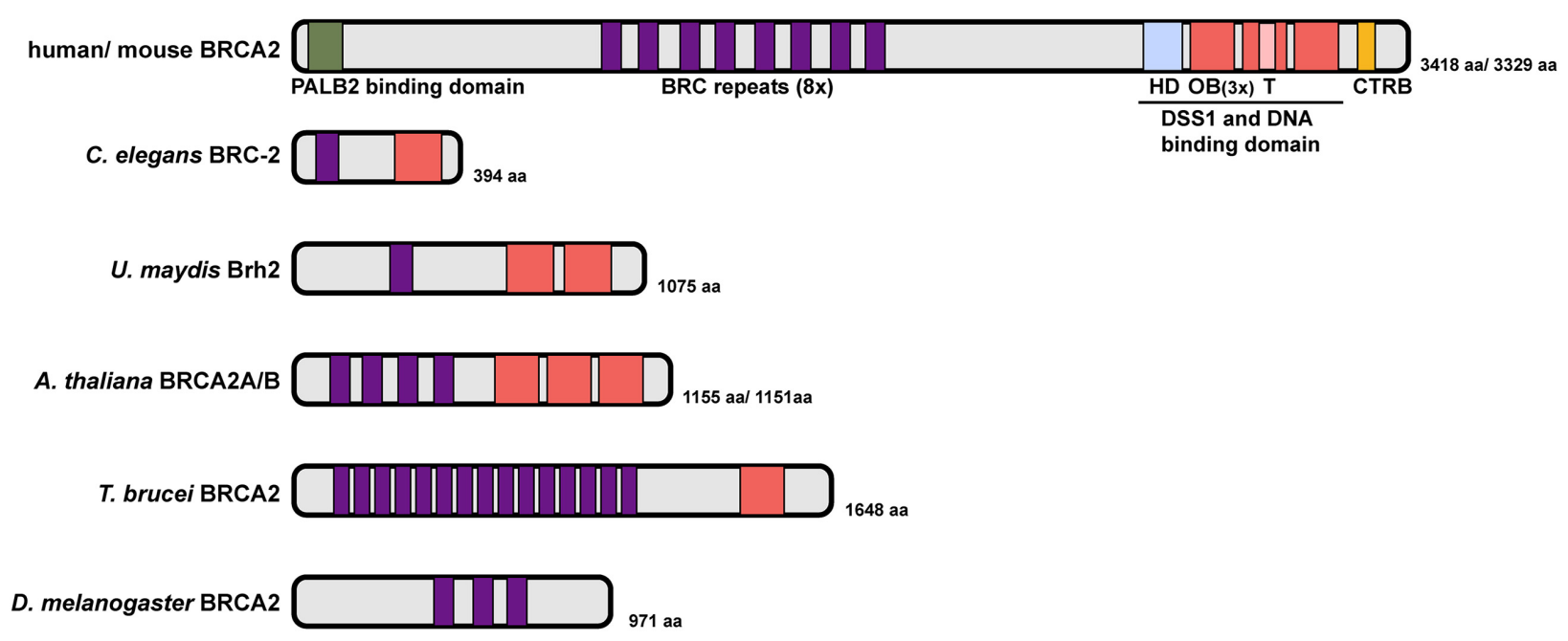

FIGURE 4 | Domain structure of BRCA2 proteins. Human BRCA2 encodes an exceptionally large protein with an N-terminal PALB2 binding domain, eight BRC repeats, a DSS1 and DNA binding domain (DBD) composed of one helix-rich domain (HD), three oligonucleotide/oligosaccharide binding (OB) folds and a tower domain, and a C terminal RAD51 binding domain (CTRB). C. elegans BRC-2 represents a simplified version with a single BRC repeat and OB fold. The number of $\mathrm{BRC}$ repeats and $\mathrm{OB}$ fold domains vary greatly in different organisms (U. maydis Brh2, A. thaliana BRCA2A/B, T. brucei BRCA2 and D. melanogaster BRCA2). Sequence alignment did not identify a putative OB fold/DNA binding domain in Drosophila BRCA2 (Yang et al., 2002).

two OB folds (Kojic et al., 2002, 2005). Drosophila melanogaster BRCA2 contains three BRC repeats but no recognizable OB fold (Klovstad et al., 2008). Two almost identical BRCA2 orthologs were identified in Arabidopsis thaliana, each containing four BRC repeats (Siaud et al., 2004). In contrast, the parasite Trypanosoma brucei possess a single BRCA2 ortholog with 15 $\mathrm{BRC}$ repeats (Hartley and McCulloch, 2008). The BRC repeat is highly conserved among species; despite the different number of repeats, BRC domains in all BRCA2 orthologs examined so far have been shown to bind RAD51 directly and to promote RAD51 nucleoprotein filament formation on ssDNA, which is essential for homology search and strand exchange during HR. In addition, BRCA2 interaction with the highly conserved DSS1 protein also contributes to HR through promoting RAD51recruitment activity and stability of BRCA2 (Li et al., 2006; Liu et al., 2010; Siaud et al., 2011). The CTRB domain, while conferring RAD51 binding and stabilizing RAD51 filaments on ssDNA, is not essential for HR (Davies and Pellegrini, 2007; Esashi et al., 2007; Prakash et al., 2015).

C. elegans BRCA2 (BRC-2) contains domain signatures similar to mammalian BRCA2 but is approximately $1 / 8$ the size, with just 394 amino acids. BRC-2 contains a single BRC repeat that directly interacts with RAD51 and a single OB fold that preferentially binds to ssDNA (Martin et al., 2005; Petalcorin et al., 2006; Figure 4). The single BRC repeat is comprised of two RAD-51 interaction regions, one that preferentially binds to free RAD51, and the other to the RAD-51-DNA nucleoprotein filament that exhibits inhibitory activity on RAD-51 ATPase hydrolysis. Together, these two RAD-51 interaction regions within the $\mathrm{BRC}$ repeat are proposed to coordinate the activity of BRC2 for promoting RAD-51 nucleation on ssDNA and stabilizing existing RAD51 filament from disassembly through inhibiting ATP hydrolysis (Petalcorin et al., 2007). Recent single-molecule analysis has revealed that BRC-2 acts primarily as a RAD-51 nucleation factor on RPA-coated ssDNA (Belan et al., 2021).

\section{BRCA2 Role in Meiotic Recombination}

In addition to a role of promoting RAD51 mediated HR in somatic cells, studies on BRCA2 orthologs have revealed a requirement for BRCA2 during meiosis. In Ustilago maydis, mutation of Brh2 led to a failure in the formation of meiotic spore products (Kojic et al., 2002). Null mutants of BRCA2 ortholog in Drosophila led to sterility in both male and female flies (Klovstad et al., 2008; Weinberg-Shukron et al., 2018). A transgenic mouse line expressing low levels of human BRCA2 in the gonad showed reduced RAD51 and DMC1 foci formation and prophase arrest of spermatocytes, due to the inability to complete meiotic recombination (Sharan et al., 2004). Depletion of $A$. thaliana BRCA2 by RNAi showed meiotic defects similar to rad51; dmc1 double mutants (Siaud et al., 2004) and C. elegans brc-2 mutant produced completely inviable progeny (Martin et al., 2005), suggesting an indispensable role of BRCA2 during meiosis. Studies on human and Arabidopsis BRCA2 proteins demonstrated that BRCA2 directly binds to the meiosis-specific recombinase DMC1, which functions together with RAD51 to promote strand invasion and joint molecule formation during meiotic recombination (Dray et al., 2006; Thorslund et al., 2007; Jensen et al., 2010; Martinez et al., 2016). As with RAD51, the BRC repeats facilitate binding between BRCA2 and DMC1, although binding affinities for each individual BRC repeat differ between RAD51 and DMC1 (Martinez et al., 2016). Moreover, different mechanisms have been proposed for BRCA2 stimulation of RAD51 versus DMC1 recombinase activity. In the context of RAD51 mediated recombination, BRCA2 and its eight BRC repeats function by a combination of inhibiting RAD51 ATPase activity, promoting RAD51 filament formation 
on ssDNA but not dsDNA, and enhancing strand exchange activity of RAD51. In contrast, stabilization of DMC1 filament on ssDNA was proposed to be the major mechanism by which BRCA2 functions with DMC1 (Martinez et al., 2016; Figure 5).

BRCA2 localization to DSBs in somatic cells depends on PALB2 (Xia et al., 2006). It has remained mysterious until recently, how BRCA2 is recruited to DSBs during meiosis. The Shibuya group identified a BRCA2 localizer in mice, which they named meiotic localizer of BRCA2 (MEILB2). MEILB2 is specifically expressed in germ cells and localizes to meiotic recombination sites on the chromosome axis. In the absence of MEILB2, the recruitment of DMC1 and RAD51 recombinase to meiotic DSBs is abolished, leading to sterility in male mice. Furthermore, MEILB2 directly binds to BRCA2 in vitro and is a physiological binding partner of BRCA2 in vivo. Removing MEILB2 impairs BRCA2 localization to resected ssDNA in spermatocytes, suggesting that MEILB2 recruits BRCA2 to sites undergoing meiotic recombination (Zhang et al., 2019). In contrast to males, female Meilb2 ${ }^{-/-}$mice show only a $\sim 50 \%$ reduction in the localization of DMC1 and RAD51, and are sub-fertile, suggesting that redundant mechanisms exist to localize BRAC2 in oogenesis. One possibility is that PALB2 functions in concert with MEILB2 in female meiosis to localize BRCA2. Interestingly, PALB2 knockout mice show reduced male, but not female, fertility. This reduction in fertility is likely due to PALB2 interaction with BRCA1 (Simhadri et al., 2014). Future studies addressing the roles, redundancies and interconnections between PALB2, BRCA1 and BRCA2 will be important for understanding how meiotic DSBs are processed in male and female meiosis. Recently a third component of the BRCA2 complex, BRCA2 and MEILB2-associating protein 1 (BRME1), was identified. BRME1 forms a ternary complex with BRCA2 and MEILB2 and in the absence of BRME1, meiotic DSB repair, homologous chromosome synapsis and crossover formation were impaired in spermatogenesis (Takemoto et al., 2020; Zhang et al., 2020). MEILB2 is conserved among vertebrate taxa; whether binding partners promote meiotic regulation of BRCA2 in organisms such as worms and plants remain to be investigated.

\section{Non-conserved Role of BRCA2 in C. elegans Meiosis}

BRCA2's role in promoting RAD51/DMC1 nucleoprotein filament formation for homology search and strand exchange in meiotic recombination is conserved among all organisms where it has been examined. A RAD-51 independent, nonconserved role of BRC-2 was uncovered in C. elegans meiosis (Martin et al., 2005; Petalcorin et al., 2006). Without BRC-2, SPO-11 induced DSBs are resected, but RAD-51 is not recruited to the single stranded DNA, blocking strand invasion for errorfree repair. As the presence of DSBs is extremely deleterious, alternative repair pathways are engaged to remove any remaining breaks before cells exit meiotic prophase I. In rad-51 or brc2 single mutant, oogenic diakinesis nuclei exhibit aggregated DAPI staining chromosome structures, in contrast to the six
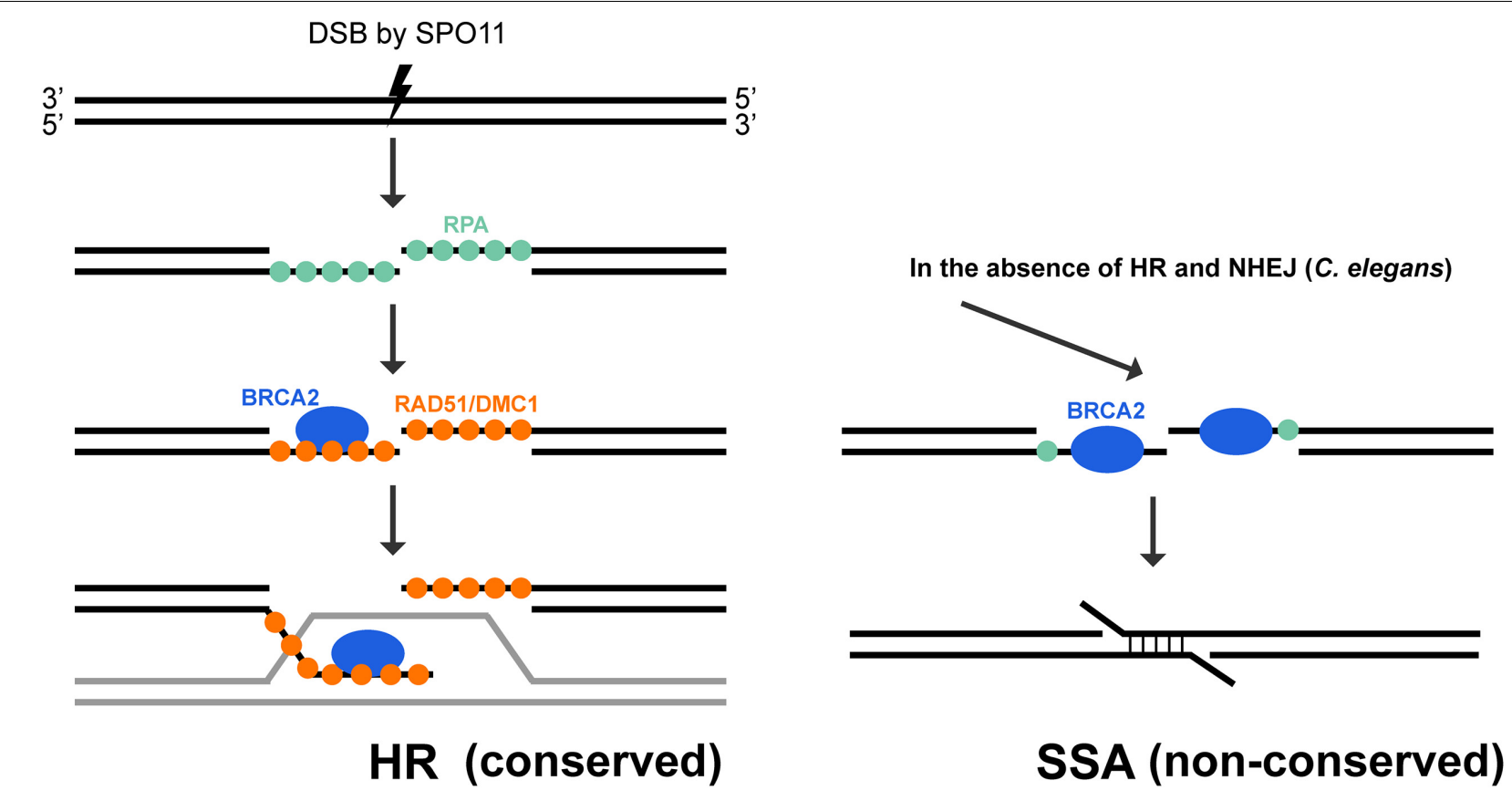

FIGURE 5 | Conserved and non-conserved roles of BRCA2 during meiosis. BRCA2 is an essential mediator of homologous recombination in meiosis. After SPO-11 induced DSB is resected, the $3^{\prime}$ ssDNA is coated with RPA. BRCA2 is critical for recruiting DMC1/RAD51 recombinases to displace RPA molecules on the ssDNA, promoting the formation and stabilization of nucleoprotein filaments to mediate homology search and strand exchange. This function of BRCA2 is highly conserved during meiosis among a large variety of organisms, including C. elegans. However, C. elegans BRC-2 also exhibits a non-conserved role in promoting single strand annealing when HR (rad-51 mutant) and NHEJ (lig-4 knock down) are not available for repair (Martin et al., 2005). 
morphologically distinct bivalent structures in wild-type animals (Figure 3B). Inactivating NHEJ (lig-4) in the brc-2 mutant resulted in mostly twelve DAPI bodies (Figure 3B), suggesting that the aggregation observed in $b r c-2$ is due to inappropriate repair of meiotic DSBs by NHEJ. However, when a functional BRC-2 was present, as in the case of the lig-4; rad-51 double mutant, diakinesis nuclei contained clumped DAPI structures as seen in $b r c-2$ and rad-51 single mutants (Figure 3B; Martin et al., 2005). This observation suggests that BRC-2 promotes an alternative repair pathway when both $\mathrm{HR}$ and NHEJ fail to be executed in meiocytes. A possible candidate for this repair pathway is single strand annealing (SSA). Indeed, in vitro experiments showed that purified C. elegans BRC-2 protein promoted annealing of single strand oligonucleotide coated with RPA (Petalcorin et al., 2006), an activity that mammalian BRCA2 does not possess (Jensen et al., 2010; Figure 5). It is likely that C. elegans BRC-2 has acquired this function to promote SSA during meiosis, as an ortholog of RAD52, which mediates SSA, is missing.

\section{CONCLUSION}

That organisms such as mice, C. elegans, and A. thaliana carrying mutations in their respective BRCA1 and BRCA2 orthologs exhibit meiotic phenotypes is consistent with BRCA1 and BRCA2 playing critical roles in meiosis. While important for meiotic recombination, BRCA1 and BRCA2 orthologs have acquired divergent functions throughout evolution. BRCA1 together with BARD1 functions as an E3 ubiquitin ligase that promotes ubiquitin transfer to a number of substrates and therefore plays regulatory roles in various processes. Not surprisingly, BRCA1 function during meiosis is quite diverse in different organisms (Figure 2). For example, BRCA1 is essential for MSCI in mice but is dispensable for MSCI in C. elegans, while C. elegans BRC-1 promotes DNA end resection, stabilizes the RAD-51 filament and regulates the crossover landscape. It remains an open question whether BRCA1-BARD1 functions in any of these aspects of meiotic recombination in mammals. Future studies taking advantage of conditional expression and genome editing tools should facilitate analyses on the role of E3 ligase activity, including identification of substrates, and the conserved BRCT domains. In contrast to BRCA1, BRCA2 plays a fundamental and conserved role in $\mathrm{HR}$ as a mediator to recruit RAD51 and DMC1 for nucleoprotein filament formation and strand

\section{REFERENCES}

Abbott, D. W., Freeman, M. L., and Holt, J. T. (1998). Double-strand break repair deficiency and radiation sensitivity in BRCA2 mutant cancer cells. J. Natl. Cancer Inst. 90, 978-985. doi: 10.1093/jnci/90.13.978

Adamo, A., Montemauri, P., Silva, N., Ward, J. D., Boulton, S. J., and La Volpe, A. (2008). BRC-1 acts in the inter-sister pathway of meiotic doublestrand break repair. EMBO Rep. 9, 287-292. doi: 10.1038/sj.embor.74 01167

Aparicio, T., Baer, R., Gottesman, M., and Gautier, J. (2016). MRN, CtIP, and BRCA1 mediate repair of topoisomerase II-DNA adducts. J. Cell Biol. 212, 399-408. doi: 10.1083/jcb.201504005 invasion. However, C. elegans BRC-2 also uniquely promotes the alternative SSA pathway, perhaps as a consequence of a streamlined set of repair proteins (e.g., absence of DMC1 and RAD52) (Figure 5). While not identical, knowledge on meiotic roles of BRCA1 and BRCA2 from model organisms will continue to provide valuable insights into the mechanisms by which these two genes function during human meiosis. Clinical data has shown a correlation between the presence of BRCA1 and BRCA2 mutations in healthy carriers and ovarian aging, which is measured by elevated accumulation of DNA damage in oocytes and reduced primordial follicle reserve (Oktay et al., 2010; Lin et al., 2017; Lambertini et al., 2018). This indicates that the functions of BRCA1 and BRCA2 during human meiosis are likely to influence sperm and egg quality. Interestingly, some cancers inappropriately express meiotic genes and recent evidence suggests that this may lead to altered BRCA2 function (Hosoya et al., 2011; Zhang et al., 2020). HR was inhibited in somatic cells when the SC protein SYCP3 and the meiotic partners of BRCA2, MEILB2 and BRME1, were aberrantly expressed, presumably as a result of BRCA2 protein being sequestered when bound by the meiotic proteins. Future studies focusing on meiotic aspects of BRCA1 and BRCA2 may advance our knowledge in human reproduction as well as tumorigenesis to provide tools for improving fertility and health.

\section{AUTHOR CONTRIBUTIONS}

QL wrote the manuscript with content and editorial input from JE. Both authors contributed to the article and approved the submitted version.

\section{FUNDING}

Work in the Engebrecht lab is supported by National Institutes of Health GM103860.

\section{ACKNOWLEDGMENTS}

We are grateful to members of the Engebrecht lab for thoughtful discussions. We also acknowledge the meiosis research community and apologize to those researchers whose work we were unable to include.

Baer, R., and Ludwig, T. (2002). The BRCA1/BARD1 heterodimer, a tumor suppressor complex with ubiquitin E3 ligase activity. Curr. Opin. Genet. Dev. 12, 86-91. doi: 10.1016/s0959-437x(01)00269-6

Baudat, F., and de Massy, B. (2007). Regulating double-stranded DNA break repair towards crossover or non-crossover during mammalian meiosis. Chromosome Res. 15, 565-577. doi: 10.1007/s10577-007-1140-3

Belan, O., Barroso, C., Kaczmarczyk, A., Anand, R., Federico, S., O’reilly, N., et al. (2021). Single-molecule analysis reveals cooperative stimulation of Rad51 filament nucleation and growth by mediator proteins. Mol. Cell 81, 1058.e71073.e7.

Boulton, S. J., Martin, J. S., Polanowska, J., Hill, D. E., Gartner, A., and Vidal, M. (2004). BRCA1/BARD1 orthologs required for DNA repair in 
Caenorhabditis elegans. Curr. Biol. 14, 33-39. doi: 10.1016/j.cub.2003. 11.029

Broering, T. J., Alavattam, K. G., Sadreyev, R. I., Ichijima, Y., Kato, Y., Hasegawa, K., et al. (2014). BRCA1 establishes DNA damage signaling and pericentric heterochromatin of the X chromosome in male meiosis. J. Cell Biol. 205, 663-675. doi: $10.1083 /$ jcb.201311050

Brown, M. S., and Bishop, D. K. (2014). DNA strand exchange and RecA homologs in meiosis. Cold Spring Harb. Perspect. Biol. 7:a016659. doi: 10.1101/ cshperspect.a016659

Brzovic, P. S., Rajagopal, P., Hoyt, D. W., King, M. C., and Klevit, R. E. (2001). Structure of a BRCA1-BARD1 heterodimeric RING-RING complex. Nat. Struct. Biol. 8, 833-837.

Bunting, S. F., Callen, E., Wong, N., Chen, H. T., Polato, F., Gunn, A., et al. (2010). 53BP1 inhibits homologous recombination in Brcal-deficient cells by blocking resection of DNA breaks. Cell 141, 243-254. doi: 10.1016/j.cell.2010. 03.012

Caestecker, K. W., and Van de Walle, G. R. (2013). The role of BRCA1 in DNA double-strand repair: past and present. Exp. Cell Res. 319, 575-587. doi: 10. 1016/j.yexcr.2012.11.013

Carreira, A., Hilario, J., Amitani, I., Baskin, R. J., Shivji, M. K., Venkitaraman, A. R., et al. (2009). The BRC repeats of BRCA2 modulate the DNA-binding selectivity of RAD51. Cell 136, 1032-1043. doi: 10.1016/j.cell.2009.02.019

Chen, C. F., Chen, P. L., Zhong, Q., Sharp, Z. D., and Lee, W. H. (1999). Expression of BRC repeats in breast cancer cells disrupts the BRCA2-Rad51 complex and leads to radiation hypersensitivity and loss of $\mathrm{G}(2) / \mathrm{M}$ checkpoint control. J. Biol. Chem. 274, 32931-32935. doi: 10.1074/jbc.274.46.32931

Chung, G., Rose, A. M., Petalcorin, M. I., Martin, J. S., Kessler, Z., Sanchez-Pulido, L., et al. (2015). REC-1 and HIM-5 distribute meiotic crossovers and function redundantly in meiotic double-strand break formation in Caenorhabditis elegans. Genes Dev. 29, 1969-1979. doi: 10.1101/gad.266056.115

Cressman, V. L., Backlund, D. C., Avrutskaya, A. V., Leadon, S. A., Godfrey, V., and Koller, B. H. (1999). Growth retardation, DNA repair defects, and lack of spermatogenesis in BRCA1-deficient mice. Mol. Cell Biol. 19, 7061-7075. doi: $10.1128 / \mathrm{mcb} \cdot 19.10 .7061$

Cruz-Garcia, A., Lopez-Saavedra, A., and Huertas, P. (2014). BRCA1 accelerates CtIP-mediated DNA-end resection. Cell Rep. 9, 451-459. doi: 10.1016/j.celrep. 2014.08.076

Daley, J. M., and Sung, P. (2014). 53BP1, BRCA1, and the choice between recombination and end joining at DNA double-strand breaks. Mol. Cell Biol. 34, 1380-1388. doi: $10.1128 / \mathrm{mcb} .01639-13$

Davies, O. R., and Pellegrini, L. (2007). Interaction with the BRCA2 C terminus protects RAD51-DNA filaments from disassembly by BRC repeats. Nat. Struct. Mol. Biol. 14, 475-483. doi: 10.1038/nsmb1251

Deng, C. X. (2002). Roles of BRCA1 in centrosome duplication. Oncogene 21, 6222-6227. doi: 10.1038/sj.onc. 1205713

Deng, C. X. (2006). BRCA1: cell cycle checkpoint, genetic instability, DNA damage response and cancer evolution. Nucleic Acids Res. 34, 1416-1426. doi: 10.1093/ nar/gkl010

Densham, R. M., Garvin, A. J., Stone, H. R., Strachan, J., Baldock, R. A., DazaMartin, M., et al. (2016). Human BRCA1-BARD1 ubiquitin ligase activity counteracts chromatin barriers to DNA resection. Nat. Struct. Mol. Biol. 23, 647-655. doi: $10.1038 / \mathrm{nsmb} .3236$

Dernburg, A. F., Mcdonald, K., Moulder, G., Barstead, R., Dresser, M., and Villeneuve, A. M. (1998). Meiotic recombination in C. elegans initiates by a conserved mechanism and is dispensable for homologous chromosome synapsis. Cell 94, 387-398. doi: 10.1016/s0092-8674(00)81481-6

Deshaies, R. J., and Joazeiro, C. A. (2009). RING domain E3 ubiquitin ligases. Аnnu. Rev. Biochem. 78, 399-434. doi: 10.1146/annurev.biochem.78.101807. 093809

Dray, E., Siaud, N., Dubois, E., and Doutriaux, M. P. (2006). Interaction between Arabidopsis Brca2 and its partners Rad51, Dmc1, and Dss1. Plant Physiol. 140, 1059-1069. doi: 10.1104/pp.105.075838

Esashi, F., Christ, N., Gannon, J., Liu, Y., Hunt, T., Jasin, M., et al. (2005). CDK-dependent phosphorylation of BRCA2 as a regulatory mechanism for recombinational repair. Nature 434, 598-604. doi: 10.1038/nature03404

Esashi, F., Galkin, V. E., Yu, X., Egelman, E. H., and West, S. C. (2007). Stabilization of RAD51 nucleoprotein filaments by the C-terminal region of BRCA2. Nat. Struct. Mol. Biol. 14, 468-474. doi: 10.1038/nsmb1245
Fernandez-Capetillo, O., Mahadevaiah, S. K., Celeste, A., Romanienko, P. J., Camerini-Otero, R. D., Bonner, W. M., et al. (2003). H2AX is required for chromatin remodeling and inactivation of sex chromosomes in male mouse meiosis. Dev. Cell 4, 497-508. doi: 10.1016/s1534-5807(03)00093-5

Fox, D. III, Le Trong, I., Rajagopal, P., Brzovic, P. S., Stenkamp, R. E., and Klevit, R. E. (2008). Crystal structure of the BARD1 ankyrin repeat domain and its functional consequences. J. Biol. Chem. 283, 21179-21186. doi: 10.1074/jbc. m802333200

Futreal, P. A., Liu, Q., Shattuck-Eidens, D., Cochran, C., Harshman, K., Tavtigian, S., et al. (1994). BRCA1 mutations in primary breast and ovarian carcinomas. Science 266, 120-122. doi: 10.1126/science.7939630

Garcia, V., Phelps, S. E., Gray, S., and Neale, M. J. (2011). Bidirectional resection of DNA double-strand breaks by Mre11 and Exol. Nature 479, 241-244. doi: $10.1038 /$ nature 10515

Garcia-Muse, T., Galindo-Diaz, U., Garcia-Rubio, M., Martin, J. S., Polanowska, J., O'reilly, N., et al. (2019). A meiotic checkpoint alters repair partner bias to permit inter-sister repair of persistent DSBs. Cell Rep. 26, 775.e5-787.e5.

Ghimenti, C., Sensi, E., Presciuttini, S., Brunetti, I. M., Conte, P., Bevilacqua, G., et al. (2002). Germline mutations of the BRCA1-associated ring domain (BARD1) gene in breast and breast/ovarian families negative for BRCA1 and BRCA2 alterations. Genes Chromosomes Cancer 33, 235-242. doi: 10.1002/gcc. 1223

Godwin, A. K., Vanderveer, L., Schultz, D. C., Lynch, H. T., Altomare, D. A., Buetow, K. H., et al. (1994). A common region of deletion on chromosome 17q in both sporadic and familial epithelial ovarian tumors distal to BRCA1. Am. J. Hum. Genet. 55, 666-677.

Gowen, L. C., Johnson, B. L., Latour, A. M., Sulik, K. K., and Koller, B. H. (1996). Brcal deficiency results in early embryonic lethality characterized by neuroepithelial abnormalities. Nat. Genet. 12, 191-194. doi: 10.1038/ng02 96-191

Gray, S., and Cohen, P. E. (2016). Control of meiotic crossovers: from doublestrand break formation to designation. Annu. Rev. Genet. 50, 175-210. doi: 10.1146/annurev-genet-120215-035111

Gudmundsdottir, K., and Ashworth, A. (2004). BRCA2 in meiosis: turning over a new leaf. Trends Cell Biol. 14, 401-404. doi: 10.1016/j.tcb.2004. 07.002

Hakem, R., De La Pompa, J. L., Sirard, C., Mo, R., Woo, M., Hakem, A., et al. (1996). The tumor suppressor gene Brcal is required for embryonic cellular proliferation in the mouse. Cell 85, 1009-1023. doi: 10.1016/s0092-8674(00) 81302-1

Hall, J. M., Lee, M. K., Newman, B., Morrow, J. E., Anderson, L. A., Huey, B., et al. (1990). Linkage of early-onset familial breast cancer to chromosome 17q21. Science 250, 1684-1689. doi: 10.1126/science.2270482

Hartley, C. L., and McCulloch, R. (2008). Trypanosoma brucei BRCA2 acts in antigenic variation and has undergone a recent expansion in BRC repeat number that is important during homologous recombination. Mol. Microbiol. 68, 1237-1251. doi: 10.1111/j.1365-2958.2008.06230.x

Hashizume, R., Fukuda, M., Maeda, I., Nishikawa, H., Oyake, D., Yabuki, Y., et al. (2001). The RING heterodimer BRCA1-BARD1 is a ubiquitin ligase inactivated by a breast cancer-derived mutation. J. Biol. Chem. 276, 14537-14540. doi: $10.1074 / j b c . c 000881200$

Hatchi, E., Skourti-Stathaki, K., Ventz, S., Pinello, L., Yen, A., Kamieniarz-Gdula, K., et al. (2015). BRCA1 recruitment to transcriptional pause sites is required for R-loop-driven DNA damage repair. Mol. Cell 57, 636-647. doi: 10.1016/j. molcel.2015.01.011

Hill, S. J., Rolland, T., Adelmant, G., Xia, X., Owen, M. S., Dricot, A., et al. (2014). Systematic screening reveals a role for BRCA1 in the response to transcriptionassociated DNA damage. Genes Dev. 28, 1957-1975. doi: 10.1101/gad.2416 20.114

Hillers, K. J., Jantsch, V., Martinez-Perez, E., and Yanowitz, J. L. (2017). Meiosis. WormBook 2017, 1-43. doi: 10.1016/b978-0-12-503365-7.50005-5

Hong, Y., Sonneville, R., Agostinho, A., Meier, B., Wang, B., Blow, J. J., et al. (2016). The SMC-5/6 complex and the HIM-6 (BLM) helicase synergistically promote meiotic recombination intermediate processing and chromosome maturation during Caenorhabditis elegans Meiosis. PLoS Genet. 12:e1005872. doi: 10.1371/ journal.pgen.1005872

Hosoya, N., Okajima, M., Kinomura, A., Fujii, Y., Hiyama, T., Sun, J., et al. (2011). Synaptonemal complex protein SYCP3 impairs mitotic recombination 
by interfering with BRCA2. EMBO Rep. 13, 44-51. doi: 10.1038/embor.20 11.221

Huber, L. J., Yang, T. W., Sarkisian, C. J., Master, S. R., Deng, C. X., and Chodosh, L. A. (2001). Impaired DNA damage response in cells expressing an exon 11deleted murine Brcal variant that localizes to nuclear foci. Mol. Cell Biol. 21, 4005-4015. doi: 10.1128/mcb.21.12.4005-4015.2001

Jagut, M., Hamminger, P., Woglar, A., Millonigg, S., Paulin, L., Mikl, M., et al. (2016). Separable roles for a Caenorhabditis elegans RMI1 homolog in promoting and antagonizing meiotic crossovers ensure faithful chromosome inheritance. PLoS Biol. 14:e1002412. doi: 10.1371/journal.pbio.10 02412

Janisiw, E., Dello Stritto, M. R., Jantsch, V., and Silva, N. (2018). BRCA1BARD1 associate with the synaptonemal complex and pro-crossover factors and influence RAD-51 dynamics during Caenorhabditis elegans meiosis. PLoS Genet. 14:e1007653. doi: 10.1371/journal.pgen.1007653

Jensen, R. B., Carreira, A., and Kowalczykowski, S. C. (2010). Purified human BRCA2 stimulates RAD51-mediated recombination. Nature 467, 678-683. doi: 10.1038 /nature09399

Kamp, J. A., Van Schendel, R., Dilweg, I. W., and Tijsterman, M. (2020). BRCA1-associated structural variations are a consequence of polymerase thetamediated end-joining. Nat. Commun. 11:3615.

King, M. C., Marks, J. H., Mandell, J. B., and New York Breast Cancer Study Group (2003). Breast and ovarian cancer risks due to inherited mutations in BRCA1 and BRCA2. Science 302, 643-646. doi: 10.1126/science.10 88759

Klovstad, M., Abdu, U., and Schupbach, T. (2008). Drosophila brca2 is required for mitotic and meiotic DNA repair and efficient activation of the meiotic recombination checkpoint. PLoS Genet. 4:e31. doi: 10.1371/journal.pgen. 0040031

Kojic, M., Kostrub, C. F., Buchman, A. R., and Holloman, W. K. (2002). BRCA2 homolog required for proficiency in DNA repair, recombination, and genome stability in Ustilago maydis. Mol. Cell 10, 683-691. doi: 10.1016/s1097-2765(02) 00632-9

Kojic, M., Zhou, Q., Lisby, M., and Holloman, W. K. (2005). Brh2-Dss1 interplay enables properly controlled recombination in Ustilago maydis. Mol. Cell Biol. 25, 2547-2557. doi: 10.1128/mcb.25.7.2547-2557.2005

Kouznetsova, A., Wang, H., Bellani, M., Camerini-Otero, R. D., Jessberger, R., and Hoog, C. (2009). BRCA1-mediated chromatin silencing is limited to oocytes with a small number of asynapsed chromosomes. J. Cell Sci. 122, 2446-2452. doi: $10.1242 /$ jcs. 049353

Kowalczykowski, S. C. (2015). An overview of the molecular mechanisms of recombinational DNA repair. Cold Spring Harb. Perspect. Biol. 7:a016410. doi: $10.1101 /$ cshperspect.a016410

Lam, I., and Keeney, S. (2014). Mechanism and regulation of meiotic recombination initiation. Cold Spring Harb. Perspect. Biol. 7:a016634. doi: 10.1101/cshperspect.a016634

Lambertini, M., Goldrat, O., Ferreira, A. R., Dechene, J., Azim, H. A. Jr., Desir, J., et al. (2018). Reproductive potential and performance of fertility preservation strategies in BRCA-mutated breast cancer patients. Ann. Oncol. 29, 237-243. doi: 10.1093/annonc/mdx639

Leon-Ortiz, A. M., Panier, S., Sarek, G., Vannier, J. B., Patel, H., Campbell, P. J., et al. (2018). A distinct class of genome rearrangements driven by heterologous recombination. Mol. Cell 69, 292.e6-305.e6.

Li, J., Zou, C., Bai, Y., Wazer, D. E., Band, V., and Gao, Q. (2006). DSS1 is required for the stability of BRCA2. Oncogene 25, 1186-1194. doi: 10.1038/ sj.onc. 1209153

Li, M. L., and Greenberg, R. A. (2012). Links between genome integrity and BRCA1 tumor suppression. Trends Biochem. Sci. 37, 418-424. doi: 10.1016/j.tibs.2012. 06.007

Li, Q., Hariri, S., and Engebrecht, J. (2020). Meiotic double-strand break processing and crossover patterning are regulated in a sex-specific manner by BRCA1BARD1 in Caenorhabditis elegans. Genetics 216, 359-379. doi: 10.1534/genetics. 120.303292

Li, Q., Saito, T. T., Martinez-Garcia, M., Deshong, A. J., Nadarajan, S., Lawrence, K. S., et al. (2018). The tumor suppressor BRCA1-BARD1 complex localizes to the synaptonemal complex and regulates recombination under meiotic dysfunction in Caenorhabditis elegans. PLoS Genet. 14:e1007701. doi: 10.1371/ journal.pgen.1007701
Lin, W., Titus, S., Moy, F., Ginsburg, E. S., and Oktay, K. (2017). Ovarian aging in women with BRCA germline mutations. J. Clin. Endocrinol. Metab. 102, 3839-3847. doi: 10.1210/jc.2017-00765

Liu, C. Y., Flesken-Nikitin, A., Li, S., Zeng, Y., and Lee, W. H. (1996). Inactivation of the mouse Brcal gene leads to failure in the morphogenesis of the egg cylinder in early postimplantation development. Genes Dev. 10, 1835-1843. doi: $10.1101 /$ gad.10.14.1835

Liu, J., Doty, T., Gibson, B., and Heyer, W. D. (2010). Human BRCA2 protein promotes RAD51 filament formation on RPA-covered single-stranded DNA. Nat. Struct. Mol. Biol. 17, 1260-1262. doi: 10.1038/nsmb.1904

Ludwig, T., Chapman, D. L., Papaioannou, V. E., and Efstratiadis, A. (1997). Targeted mutations of breast cancer susceptibility gene homologs in mice: lethal phenotypes of Brca1, Brca2, Brca1/Brca2, Brca1/p53, and Brca2/p53 nullizygous embryos. Genes Dev. 11, 1226-1241. doi: 10.1101/gad.11.10.1226

Lui, D. Y., and Colaiacovo, M. P. (2013). Meiotic development in Caenorhabditis elegans. Adv. Exp. Med. Biol. 757, 133-170. doi: 10.1007/978-1-4614-4015-4_6

Mahadevaiah, S. K., Bourc'his, D., De Rooij, D. G., Bestor, T. H., Turner, J. M., and Burgoyne, P. S. (2008). Extensive meiotic asynapsis in mice antagonises meiotic silencing of unsynapsed chromatin and consequently disrupts meiotic sex chromosome inactivation. J. Cell Biol. 182, 263-276. doi: 10.1083/jcb. 200710195

Manke, I. A., Lowery, D. M., Nguyen, A., and Yaffe, M. B. (2003). BRCT repeats as phosphopeptide-binding modules involved in protein targeting. Science 302, 636-639. doi: 10.1126/science.1088877

Martin, J. S., Winkelmann, N., Petalcorin, M. I., Mcilwraith, M. J., and Boulton, S. J. (2005). RAD-51-dependent and -independent roles of a Caenorhabditis elegans BRCA2-related protein during DNA double-strand break repair. Mol. Cell Biol. 25, 3127-3139. doi: 10.1128/mcb.25.8.3127-3139.2005

Martinez, J. S., Von Nicolai, C., Kim, T., Ehlen, A., Mazin, A. V., Kowalczykowski, S. C., et al. (2016). BRCA2 regulates DMC1-mediated recombination through the BRC repeats. Proc. Natl. Acad. Sci. U.S.A. 113, 3515-3520. doi: 10.1073/ pnas. 1601691113

McCarthy, E. E., Celebi, J. T., Baer, R., and Ludwig, T. (2003). Loss of Bard1, the heterodimeric partner of the Brcal tumor suppressor, results in early embryonic lethality and chromosomal instability. Mol. Cell Biol. 23, 5056-5063. doi: 10. 1128/mcb.23.14.5056-5063.2003

Meneely, P. M., Mcgovern, O. L., Heinis, F. I., and Yanowitz, J. L. (2012). Crossover distribution and frequency are regulated by him-5 in Caenorhabditis elegans. Genetics 190, 1251-1266. doi: 10.1534/genetics.111.137463

Meza, J. E., Brzovic, P. S., King, M. C., and Klevit, R. E. (1999). Mapping the functional domains of BRCA1. Interaction of the ring finger domains of BRCA1 and BARD1. J. Biol. Chem. 274, 5659-5665.

Miki, Y., Swensen, J., Shattuck-Eidens, D., Futreal, P. A., Harshman, K., Tavtigian, S., et al. (1994). A strong candidate for the breast and ovarian cancer susceptibility gene BRCA1. Science 266, 66-71. doi: 10.1126/science.7545954

Moynahan, M. E., Chiu, J. W., Koller, B. H., and Jasin, M. (1999). Brcal controls homology-directed DNA repair. Mol. Cell 4, 511-518. doi: 10.1016/s10972765(00)80202-6

Moynahan, M. E., Pierce, A. J., and Jasin, M. (2001). BRCA2 is required for homology-directed repair of chromosomal breaks. Mol. Cell 7, 263-272. doi: 10.1016/s1097-2765(01)00174-5

Nakamura, K., Saredi, G., Becker, J. R., Foster, B. M., Nguyen, N. V., Beyer, T. E., et al. (2019). H4K20me0 recognition by BRCA1-BARD1 directs homologous recombination to sister chromatids. Nat. Cell Biol. 21, 311-318. doi: 10.1038/ s41556-019-0282-9

Neale, M. J., and Keeney, S. (2006). Clarifying the mechanics of DNA strand exchange in meiotic recombination. Nature 442, 153-158. doi: 10.1038/ nature 04885

Negrini, S., Gorgoulis, V. G., and Halazonetis, T. D. (2010). Genomic instabilityan evolving hallmark of cancer. Nat. Rev. Mol. Cell Biol. 11, 220-228. doi: $10.1038 / \mathrm{nrm} 2858$

Oktay, K., Kim, J. Y., Barad, D., and Babayev, S. N. (2010). Association of BRCA1 mutations with occult primary ovarian insufficiency: a possible explanation for the link between infertility and breast/ovarian cancer risks. J. Clin. Oncol. 28, 240-244. doi: 10.1200/jco.2009.24.2057

Paiano, J., Wu, W., Yamada, S., Sciascia, N., Callen, E., Paola Cotrim, A., et al. (2020). ATM and PRDM9 regulate SPO11-bound recombination intermediates during meiosis. Nat. Commun. 11:857. 
Petalcorin, M. I., Galkin, V. E., Yu, X., Egelman, E. H., and Boulton, S. J. (2007). Stabilization of RAD-51-DNA filaments via an interaction domain in Caenorhabditis elegans BRCA2. Proc. Natl. Acad. Sci. U.S.A. 104, 8299-8304. doi: 10.1073/pnas.0702805104

Petalcorin, M. I., Sandall, J., Wigley, D. B., and Boulton, S. J. (2006). CeBRC2 stimulates D-loop formation by RAD-51 and promotes DNA single-strand annealing. J Mol. Biol. 361, 231-242. doi: 10.1016/j.jmb.2006.06.020

Polanowska, J., Martin, J. S., Garcia-Muse, T., Petalcorin, M. I., and Boulton, S. J. (2006). A conserved pathway to activate BRCA1-dependent ubiquitylation at DNA damage sites. EMBO J. 25, 2178-2188. doi: 10.1038/sj.emboj.7601102

Prakash, R., Zhang, Y., Feng, W., and Jasin, M. (2015). Homologous recombination and human health: the roles of BRCA1, BRCA2, and associated proteins. Cold Spring Harb. Perspect. Biol. 7:a016600. doi: 10.1101/cshperspect.a016600

Reidt, W., Wurz, R., Wanieck, K., Chu, H. H., and Puchta, H. (2006). A homologue of the breast cancer-associated gene BARD1 is involved in DNA repair in plants. EMBO J. 25, 4326-4337. doi: 10.1038/sj.emboj.7601313

Rodriguez, M., Yu, X., Chen, J., and Songyang, Z. (2003). Phosphopeptide binding specificities of BRCA1 COOH-terminal (BRCT) domains. J. Biol. Chem. 278, 52914-52918. doi: 10.1074/jbc.c300407200

Roy, R., Chun, J., and Powell, S. N. (2011). BRCA1 and BRCA2: different roles in a common pathway of genome protection. Nat. Rev. Cancer 12, 68-78. doi: $10.1038 / \mathrm{nrc} 3181$

Saito, T. T., and Colaiacovo, M. P. (2017). Regulation of crossover frequency and distribution during meiotic recombination. Cold Spring Harb. Symp. Quant. Biol. 82, 223-234. doi: 10.1101/sqb.2017.82.034132

Saito, T. T., Lui, D. Y., Kim, H. M., Meyer, K., and Colaiacovo, M. P. (2013). Interplay between structure-specific endonucleases for crossover control during Caenorhabditis elegans meiosis. PLoS Genet. 9:e1003586. doi: 10.1371/journal. pgen.1003586

Saito, T. T., Mohideen, F., Meyer, K., Harper, J. W., and Colaiacovo, M. P. (2012). SLX-1 is required for maintaining genomic integrity and promoting meiotic noncrossovers in the Caenorhabditis elegans germline. PLoS Genet. 8:e1002888. doi: 10.1371/journal.pgen.1002888

Scully, R., Chen, J., Plug, A., Xiao, Y., Weaver, D., Feunteun, J., et al. (1997). Association of BRCA1 with Rad51 in mitotic and meiotic cells. Cell 88, 265-275. doi: 10.1016/s0092-8674(00)81847-4

Sharan, S. K., Morimatsu, M., Albrecht, U., Lim, D. S., Regel, E., Dinh, C., et al. (1997). Embryonic lethality and radiation hypersensitivity mediated by Rad51 in mice lacking Brca2. Nature 386, 804-810. doi: 10.1038/386804a0

Sharan, S. K., Pyle, A., Coppola, V., Babus, J., Swaminathan, S., Benedict, J., et al. (2004). BRCA2 deficiency in mice leads to meiotic impairment and infertility. Development 131, 131-142. doi: 10.1242/dev.00888

Shinohara, A., and Shinohara, M. (2004). Roles of RecA homologues Rad51 and Dmcl during meiotic recombination. Cytogenet. Genome Res. 107, 201-207. doi: $10.1159 / 000080598$

Shivji, M. K., Mukund, S. R., Rajendra, E., Chen, S., Short, J. M., Savill, J., et al. (2009). The BRC repeats of human BRCA2 differentially regulate RAD51 binding on single- versus double-stranded DNA to stimulate strand exchange. Proc. Natl. Acad. Sci. U.S.A. 106, 13254-13259. doi: 10.1073/pnas.0906208106

Siaud, N., Barbera, M. A., Egashira, A., Lam, I., Christ, N., Schlacher, K., et al. (2011). Plasticity of BRCA2 function in homologous recombination: genetic interactions of the PALB2 and DNA binding domains. PLoS Genet. 7:e1002409. doi: 10.1371/journal.pgen.1002409

Siaud, N., Dray, E., Gy, I., Gerard, E., Takvorian, N., and Doutriaux, M. P. (2004). Brca2 is involved in meiosis in Arabidopsis thaliana as suggested by its interaction with Dmc1. EMBO J. 23, 1392-1401. doi: 10.1038/sj.emboj.7600146

Simhadri, S., Peterson, S., Patel, D. S., Huo, Y., Cai, H., Bowman-Colin, C., et al. (2014). Male fertility defect associated with disrupted BRCA1-PALB2 interaction in mice. J. Biol. Chem. 289, 24617-24629. doi: 10.1074/jbc.m114. 566141

Steinfeld, J. B., Belan, O., Kwon, Y., Terakawa, T., Al-Zain, A., Smith, M. J., et al. (2019). Defining the influence of Rad51 and Dmcl lineage-specific amino acids on genetic recombination. Genes Dev. 33, 1191-1207. doi: 10.1101/gad.3280 62.119

Takemoto, K., Tani, N., Takada-Horisawa, Y., Fujimura, S., Tanno, N., Yamane, M., et al. (2020). Meiosis-Specific C19orf57/4930432K21Rik/BRME1 Modulates Localization of RAD51 and DMC1 to DSBs in mouse meiotic recombination. Cell Rep. 31:107686. doi: 10.1016/j.celrep.2020.107686
Thai, T. H., Du, F., Tsan, J. T., Jin, Y., Phung, A., Spillman, M. A., et al. (1998). Mutations in the BRCA1-associated RING domain (BARD1) gene in primary breast, ovarian and uterine cancers. Hum. Mol. Genet. 7, 195-202. doi: 10.1093/ hmg/7.2.195

Thakur, S., Zhang, H. B., Peng, Y., Le, H., Carroll, B., Ward, T., et al. (1997). Localization of BRCA1 and a splice variant identifies the nuclear localization signal. Mol. Cell Biol. 17, 444-452. doi: 10.1128/mcb.17.1.444

Thorslund, T., Esashi, F., and West, S. C. (2007). Interactions between human BRCA2 protein and the meiosis-specific recombinase DMC1. EMBO J. 26, 2915-2922. doi: 10.1038/sj.emboj.7601739

Thorslund, T., Mcilwraith, M. J., Compton, S. A., Lekomtsev, S., Petronczki, M., Griffith, J. D., et al. (2010). The breast cancer tumor suppressor BRCA2 promotes the specific targeting of RAD51 to single-stranded DNA. Nat. Struct. Mol. Biol. 17, 1263-1265. doi: 10.1038/nsmb.1905

Turner, J. M. (2007). Meiotic sex chromosome inactivation. Development 134, 1823-1831. doi: 10.1242/dev.000018

Turner, J. M., Aprelikova, O., Xu, X., Wang, R., Kim, S., Chandramouli, G. V., et al. (2004). BRCA1, histone H2AX phosphorylation, and male meiotic sex chromosome inactivation. Curr. Biol. 14, 2135-2142. doi: 10.1016/j.cub.2004. 11.032

Tutt, A., Gabriel, A., Bertwistle, D., Connor, F., Paterson, H., Peacock, J., et al. (1999). Absence of Brca2 causes genome instability by chromosome breakage and loss associated with centrosome amplification. Curr. Biol. 9, 1107-1110. doi: 10.1016/s0960-9822(99)80479-5

Venkitaraman, A. R. (2002). Cancer susceptibility and the functions of BRCA1 and BRCA2. Cell 108, 171-182. doi: 10.1016/s0092-8674(02)00615-3

Volkova, N. V., Meier, B., Gonzalez-Huici, V., Bertolini, S., Gonzalez, S., Vohringer, H., et al. (2020). Mutational signatures are jointly shaped by DNA damage and repair. Nat. Commun. 11:2169.

Wagner, C. R., Kuervers, L., Baillie, D. L., and Yanowitz, J. L. (2010). xnd-1 regulates the global recombination landscape in Caenorhabditis elegans. Nature 467, 839-843. doi: 10.1038/nature09429

Weinberg-Shukron, A., Rachmiel, M., Renbaum, P., Gulsuner, S., Walsh, T., Lobel, O., et al. (2018). Essential role of BRCA2 in ovarian development and function. N. Engl. J. Med. 379, 1042-1049.

Wong, A. K., Pero, R., Ormonde, P. A., Tavtigian, S. V., and Bartel, P. L. (1997). RAD51 interacts with the evolutionarily conserved BRC motifs in the human breast cancer susceptibility gene brca2. J. Biol. Chem. 272, 31941-31944. doi: 10.1074/jbc.272.51.31941

Wooster, R., Bignell, G., Lancaster, J., Swift, S., Seal, S., Mangion, J., et al. (1995). Identification of the breast cancer susceptibility gene BRCA2. Nature 378, 789-792.

Wu, L. C., Wang, Z. W., Tsan, J. T., Spillman, M. A., Phung, A., Xu, X. L., et al. (1996). Identification of a RING protein that can interact in vivo with the BRCA1 gene product. Nat. Genet. 14, 430-440. doi: 10.1038/ng1296-430

Xia, B., Sheng, Q., Nakanishi, K., Ohashi, A., Wu, J., Christ, N., et al. (2006). Control of BRCA2 cellular and clinical functions by a nuclear partner. PALB2. Mol. Cell 22, 719-729. doi: 10.1016/j.molcel.2006.05.022

Xu, X., Aprelikova, O., Moens, P., Deng, C. X., and Furth, P. A. (2003). Impaired meiotic DNA-damage repair and lack of crossing-over during spermatogenesis in BRCA1 full-length isoform deficient mice. Development 130, 2001-2012. doi: 10.1242/dev.00410

Xu, X., Qiao, W., Linke, S. P., Cao, L., Li, W. M., Furth, P. A., et al. (2001). Genetic interactions between tumor suppressors Brcal and p53 in apoptosis, cell cycle and tumorigenesis. Nat. Genet. 28, 266-271. doi: 10.1038/90108

Xu, X., Weaver, Z., Linke, S. P., Li, C., Gotay, J., Wang, X. W., et al. (1999). Centrosome amplification and a defective G2-M cell cycle checkpoint induce genetic instability in BRCA1 exon 11 isoform-deficient cells. Mol. Cell 3, 389-395. doi: 10.1016/s1097-2765(00)80466-9

Yang, H., Jeffrey, P. D., Miller, J., Kinnucan, E., Sun, Y., Thoma, N. H., et al. (2002). BRCA2 function in DNA binding and recombination from a BRCA2DSS1-ssDNA structure. Science 297, 1837-1848. doi: 10.1126/science.297.5588. 1837

Yarden, R. I., Pardo-Reoyo, S., Sgagias, M., Cowan, K. H., and Brody, L. C. (2002). BRCA1 regulates the G2/M checkpoint by activating Chk1 kinase upon DNA damage. Nat. Genet. 30, 285-289. doi: 10.1038/ng837

Yokoo, R., Zawadzki, K. A., Nabeshima, K., Drake, M., Arur, S., and Villeneuve, A. M. (2012). COSA-1 reveals robust homeostasis and separable licensing and 
reinforcement steps governing meiotic crossovers. Cell 149, 75-87. doi: 10. 1016/j.cell.2012.01.052

Yu, V. P., Koehler, M., Steinlein, C., Schmid, M., Hanakahi, L. A., Van Gool, A. J., et al. (2000). Gross chromosomal rearrangements and genetic exchange between nonhomologous chromosomes following BRCA2 inactivation. Genes Dev. 14, 1400-1406.

Yu, X., Chini, C. C., He, M., Mer, G., and Chen, J. (2003). The BRCT domain is a phospho-protein binding domain. Science 302, 639-642. doi: 10.1126/science. 1088753

Yu, Z., Kim, Y., and Dernburg, A. F. (2016). Meiotic recombination and the crossover assurance checkpoint in Caenorhabditis elegans. Semin. Cell Dev. Biol. 54, 106-116. doi: 10.1016/j.semcdb.2016.03.014

Yuan, S. S., Lee, S. Y., Chen, G., Song, M., Tomlinson, G. E., and Lee, E. Y. (1999). BRCA2 is required for ionizing radiation-induced assembly of Rad51 complex in vivo. Cancer Res. 59, 3547-3551.

Zetka, M. C., and Rose, A. M. (1995). Mutant rec-1 eliminates the meiotic pattern of crossing over in Caenorhabditis elegans. Genetics 141, 1339-1349. doi: 10. 1093/genetics/141.4.1339

Zhang, J., Fujiwara, Y., Yamamoto, S., and Shibuya, H. (2019). A meiosis-specific BRCA2 binding protein recruits recombinases to DNA double-strand breaks to ensure homologous recombination. Nat. Commun. 10:722.
Zhang, J., Gurusaran, M., Fujiwara, Y., Zhang, K., Echbarthi, M., Vorontsov, E., et al. (2020). The BRCA2-MEILB2-BRME1 complex governs meiotic recombination and impairs the mitotic BRCA2-RAD51 function in cancer cells. Nat. Commun. 11:2055.

Zhao, W., Steinfeld, J. B., Liang, F., Chen, X., Maranon, D. G., Jian Ma, C., et al. (2017). BRCA1-BARD1 promotes RAD51-mediated homologous DNA pairing. Nature 550, 360-365. doi: 10.1038/nature24060

Zickler, D., and Kleckner, N. (2015). Recombination, pairing, and synapsis of homologs during meiosis. Cold Spring Harb. Perspect. Biol. 7:a016626. doi: 10.1101/cshperspect.a016626

Conflict of Interest: The authors declare that the research was conducted in the absence of any commercial or financial relationships that could be construed as a potential conflict of interest.

Copyright (c) $2021 \mathrm{Li}$ and Engebrecht. This is an open-access article distributed under the terms of the Creative Commons Attribution License (CC BY). The use, distribution or reproduction in other forums is permitted, provided the original author(s) and the copyright owner(s) are credited and that the original publication in this journal is cited, in accordance with accepted academic practice. No use, distribution or reproduction is permitted which does not comply with these terms. 